\title{
Cloning and expression of a novel a-1,3-arabinofuranosidase from Penicillium oxalicum sp. 68
}

\author{
Yanbo Hu, Xuecui Yan, Han Zhang, Jiaqi Liu, Feng Luo, Yingying Cui, Weiyang Wang and Yifa Zhou*
}

\begin{abstract}
The discovery and creation of biocatalysts for plant biomass conversion are essential for industrial demand and scientific research of the plant cell wall. a-1,2 and a-1,3-L-arabinofuranosidases are debranching enzymes that catalyzing hydrolytic release of a-L-arabinofuranosyl residues in plant cell wall. Gene database analyses shows that GH62 family only contains specific $a$-L-arabinofuranosidases that play an important role in the degradation and structure of the plant cell wall. At present, there are only 22 enzymes in this group has been characterized. In this study, we cloned a novel a-1,3-arabinofuranosidase gene (poabf62a) belonging to glycoside hydrolase family 62 from Penicillium oxalicum sp. 68 and expressed it in Pichia pastoris. The molecular mass of recombinant PoAbf62A was estimated to be $32.9 \mathrm{kDa}$. Using $p$-nitrophenyl-a-l-arabinofuranoside ( $p N P a A b f)$ as substrate, purified PoAbf62A exhibited an optimal $\mathrm{pH}$ of 4.5 and temperature of $35^{\circ} \mathrm{C}$. Results of methylation and ${ }^{13} \mathrm{C}$ NMR analyses showed that PoAbf62A was exclusively a-1,3-arabinofuranosidase, specific for cleavage of a-1,3-arabinofuranosyl residues, and with the absence of activity towards a-1,2-arabinofuranose and a-1,5-arabinofuranose. Therefore, PoAbf62A exhibits high activity on sugar beet arabinan and wheat arabinoxylan, because their branched side chain are decorated with a-1,3-arabinofuranose. On the other hand, there is a lack of activity with linear-a-L-1,5-arabinan and xylan that only contained a-L-1,5arabinofuranose or $\beta-1,4-x y l o s e$. The $a-1,3$-arabinofuranosidase activity identified here provides a new biocatalytic tool to degrade hemicellulose and analyze the structure of plant cell walls.
\end{abstract}

Keywords: a-L-Arabinofuranosidase, Glycoside hydrolase family 62, Penicillium oxalicum sp. 68, Sugar beet arabinan, Arabinoxylan

\section{Introduction}

Glycoside hydrolases are a broad group of enzymes that hydrolyse the glycosidic bond of saccharides and their derivatives (Scigelova et al. 1999). Presently, about 148 glycoside hydrolase families have been reported, based on sequence and structural similarities in the CAZy database (Lombard et al. 2014). There are widely used in the bio-processing industry and for the structural analysis of polysaccharides from the plant cell wall because of high efficiency, specificity, and low pollution (Himmel et al. 2007). The $\alpha$-L-arabinofuranosidases (ABFs) are

\footnotetext{
*Correspondence: zhouyf383@nenu.edu.cn

Jilin Province Key Laboratory on Chemistry and Biology of Natural

Drugs in Changbai Mountain, School of Life Sciences, Northeast Normal

University, Changchun 130024, China
}

enzymes that hydrolyze terminal non-reducing end $\alpha-\mathrm{L}-$ arabinofuranoside residues in $\alpha$ - $\mathrm{L}$-arabinosides. They are classified into families GH2, GH3, GH10, GH43, GH51, GH54 and GH62, with the ABFs of families GH43 and GH62 belonging to GH-F that have a five-bladed $\beta$ propeller arrangement and an inverting mechanism of hydrolysis (Vandermarliere et al. 2009; Wang et al. 2014; Maehara et al. 2014). The GH62 family only contains $\alpha-\mathrm{L}$-arabinofuranosidases of fungal and bacterial origin. Based on ABFs' substrate specificity, they can be divided into type A and B (Pitson et al. 1996). ABFs of type A only act on arabinoxylooligosaccharides and $p$-nitrophyl- $\alpha-\mathrm{L}-$ arabinofuranoside, whereas ABFs of type B have equivalent activities on oligosaccharide and polysaccharides (Van Laere et al. 1997). All GH62 family belong to type B, because they are more active on polymeric arabinoxylan 
that is singly or doubly substituted with $\alpha$-L-1,2-Araf and $\alpha-\mathrm{L}-1,3-$ Araf residues (Kaur et al. 2015; Hashimoto et al. 2011). They have little activity on low molecular-weight substrates, such as unbranch arabinan and $p$ NP $\alpha$ Araf (Wilkens et al. 2017; Sakamoto et al. 2011). ABFs have become known from their various industrial applications, such as biotransformation of plant residues, food processing, and bleaching of pulp (Saha 2000; Numan and Bhosle 2006). Aside from this, ABFs can completely degrade hemicelluloses and pectins in combination with other accessory hemicellulases and pectic enzymes (Margolles-Clark et al. 1996; Spagna et al. 1998). The ABFs from GH62 family are promising candidates for use in the structural analysis of polysaccharides and in regulating the chemical xylan to saccharify xylan for bio-refinery applications.

Other than D-xylose, L-arabinose is the second most abundant pentose in polysaccharides from the plant cell wall that is widely populated with arabinan, arabinoxylan, arabinogalactan and rhamnogalacturonan I (Seiboth and Metz 2011; Caffall and Mohnen 2009). The backbones of these polysaccharides often contain arabinofuranose side chains. Arabinan is composed of an $\alpha-1,5-\mathrm{L}-$ arabinofuranose backbone with $\alpha-1,3-\mathrm{L}$-arabinofuranose and $\alpha-1,2-\mathrm{L}$-arabinofuranose side chains (Sakamoto and Kawasaki 2003). Arabinoxylan consists of $\beta-1,4-$ $D$-xylan in which arabinose residues are substituted of O-2 or O-3 sites of the xylan backbone (Kormelink et al. 1993). Arabinogalactan is present on the backbone of $\beta$-1,3-galactose-linked with $\alpha$-L-arabinofuranose and $\beta$-L-arabinopyranose side chains (Ponder and Richards 1997). In addition, the rhamnogalacturonan-I backbone is also substituted with various oligosaccharides, such as arabinooligosaccharides and galactan oligosaccharides (Gruppen et al. 1993). L-Arabinose is an important sugar in the food and biofuel industries, as well as in pharmaceutics (Amore et al. 2012), and it has excellent potential as prebiotics and inhibition of sucrose absorption (Altamimi et al. 2006; Seri et al. 1996). The production of $\mathrm{L}$-arabinose by $\alpha$-L-arabinofuranosidases is efficient and environmentally friendly. As the main component of hemicellulose, xylan also has potential applications in food industry. Because the side chains of xylan are often decorated with $\alpha$-L-arabinofuranose (Song et al. 2012), it is necessary to hydrolyze arabinose residues efficiently using $\alpha$ - $\mathrm{L}$-arabinofuranosidases to produce xylan (Gao et al. 2011). Thus, it is essential to obtain a variety of $\alpha-\mathrm{L}-$ arabinofuranosidases to prepare oligosaccharide fragments and analyze the structures of polysaccharides. In this work, we have explored the biochemical characterization of a novel $\alpha-1,3$-arabinofuranosidase (PoAbf62A) from $P$. oxalicum sp. 68 , that can specially remove $\alpha-\mathrm{L}-$ 1,3-Araf from sugar beet arabinan. This work provides a new tool for industrial preparation of polysaccharides, as well as for the structural analysis of polysaccharides.

\section{Materials and methods \\ Strains and reagents}

Penicillium oxalicum sp. 68 with the collection number CGMCC 7.328 in China General Microbiological Culture Collection Center was isolated from Chang bai mountain soil (Jilin Province, China). DH5 $\alpha$, E. coli BL 21 (DE3), P. pastoris GS115, pET-32a (+) and pPIC9K (Novagen, Madison, WI, USA) were used as host and expression vectors, respectively. $p \mathrm{NP}-\alpha-\mathrm{L}$-arabinofuranoside ( $p$ NP $\alpha$ Araf $), \quad p N \mathrm{~N}-\alpha$-L-arabinopyranoside $(p$ NP $\alpha$ Ara $p)$ $p \mathrm{NP}-\beta$-galactopyranoside $\quad(p \mathrm{NP} \beta \mathrm{Gal}), \quad p \mathrm{NP}-\alpha-$ galactopyranoside ( $p \mathrm{NP} \alpha \mathrm{Gal}), \quad p \mathrm{NP}-\beta$-xylopyranoside ( $p \mathrm{NP} \beta \mathrm{Xyl}), p$-nitrophenyl- $\beta$-glucopyranoside ( $p \mathrm{NP} \beta \mathrm{Glc})$, $p \mathrm{NP}-\alpha$-glucopyranoside $(p \mathrm{NP} \alpha \mathrm{Glc})$ were purchased from Sigma (St. Louis, MO, USA). Sugar beet L-arabinan, linear-1,5- $\alpha$-L-arabinan, and wheat arabinoxylan (low viscosity) were obtained from Megazyme International Ireland Ltd. (Wicklow, Ireland). All of other chemicals and reagents were analytical grade.

\section{Construction of plasmids and strains}

The total RNA was extracted from P. oxalicum sp. 68, the mycelia were frozen in liquid nitrogen, homogenized with mortar and pestle, and extracted with Trizol. The cDNA encoding for $\alpha-1,3$-arabinofuranosidase was reverse by reverse-transcriptase-PCR using a reverse-transcriptase (Cat, No, M531A, Promega) and oligo-dT primer. According to the amino acid sequence which was reported in a previous paper (Liu et al. 2013; $\mathrm{Li}$ et al. 2013), primers having restriction sites for NdeI (GGAATTCCATATGGCTGGTACCCTTGCGAG) and EcoRI (CGGAATTCTTACTAGTGCTTCAGGGT GAGA) were used for gene amplification. PCR was performed using DreamTaq Green PCR Master Mix (Thermo Scientific), and the program was following: $95{ }^{\circ} \mathrm{C}$ for $30 \mathrm{~s}, 30$ cycles of $95^{\circ} \mathrm{C}$ for $10 \mathrm{~s}, 66^{\circ} \mathrm{C}$ for $45 \mathrm{~s}, 72{ }^{\circ} \mathrm{C}$ for $1 \mathrm{~min} 30 \mathrm{~s}$, and finally extension at $72{ }^{\circ} \mathrm{C}$ for $10 \mathrm{~min}$. The PCR product and pET-32a (+) were digested with NdeI and EcoRI, and gene was ligated with pET-32a $(+)$ to generate the recombinant plasmid pET32a-poabf62a. All enzymes used were from New England Biolab (Beverly, MA). The restriction enzyme digestions, ligations and transformations were performed as the suppliers' recommendations. For PoAbf62A expression, E. coli BL21 (DE3) cells harboring pET32a-poabf62a were grown in $100 \mathrm{ml}$ of LB broth with $100 \mu \mathrm{g} / \mathrm{ml}$ ampicillin at $37{ }^{\circ} \mathrm{C}$. When the $\mathrm{OD}_{600 \mathrm{~nm}}$ reached 0.5 , the culture was induced with $0.5 \mathrm{mM}$ IPTG and then grown for $16 \mathrm{~h}$ at $25{ }^{\circ} \mathrm{C}$. For eukaryotic expression system, the specific primers were poabf62a-F 
(GACCTACGTAGCTGGTACCCTTGCGAGTGC) and poabf62a-R (CGGAATTCTTACTAGTGCTTCAGGGTGAGA), under the following conditions: denaturing at $94{ }^{\circ} \mathrm{C}$ for $30 \mathrm{~s}$, annealing at $62{ }^{\circ} \mathrm{C}$ for $45 \mathrm{~s}$, and amplification at $72{ }^{\circ} \mathrm{C}$ for $1 \mathrm{~min} 30 \mathrm{~s}, 30$ cycles. The product of PCR was ligated with pPIC9K (SnaBI/EcoRI). Approximately $5 \mu \mathrm{g}$ of the recombinant plasmid (poabf62apPIC9K) was linearized by SalI and electrotransformed into GS115 cells. Electroporation and selection of transformants were carried out by using MD and G418. The selected clone was cultured in BGMY medium at $30{ }^{\circ} \mathrm{C}$ for 3 days, with methanol being supplemented (0.5\%) every $24 \mathrm{~h}$ during the induction period. Cells were harvested by centrifugation at $8000 \mathrm{rpm}$ for $10 \mathrm{~min}$, and the crude enzyme was found in the supernatant.

\section{Purification of recombinant PoAbf62A}

PoAbf62A was purified from the culture supernatant of recombinant $P$. pastoris GS115, and enzyme activity was assayed throughout the purification steps. The culture medium $(200 \mathrm{ml})$ was centrifuged at $8000 \mathrm{rpm}$ for $10 \mathrm{~min}$, and the supernatant collected and precipitated with $80 \%$ ammonium sulfate, dissolved and dialyzed against $20 \mathrm{mM} \mathrm{Na}$-acetate buffer ( $\mathrm{pH} 4.5)$. The protein was passed through a $10 \times 1.6 \mathrm{~cm}$ Phenyl Sepharose 6 Fast Flow (high sub) column (GE Healthcare) which contained ammonium sulfate at a saturated concentration of $30 \%$. Adsorbed proteins were then eluted by gradient elution with ammonium sulfate (from $30,20,10,0 \%$ of saturation) at a flow rate of $2 \mathrm{ml} / \mathrm{min}$, and each gradient was eluted with two fold column volume of buffer. The purified PoAbf62A was analyzed by sodium dodecyl sulfate-polyacrylamide gel electrophoresis (SDS-PAGE) on 10\% separating gel (Laemmli 1970), and protein concentrations were determined by using the method of Bradford with bovine serum albumin (BSA) as the standard (Bradford 1976).

\section{Biochemical characterization}

Specific activity was measured in $200 \mu \mathrm{l}$ of $20 \mathrm{mM} \mathrm{Na}-$ acetate buffer ( $\mathrm{pH} 4.5)$ containing $10 \mathrm{mM} p \mathrm{NP} \alpha \mathrm{Ara} f$ and $2 \mu \mathrm{g}$ recombinant PoAbf62A. After incubating at $37^{\circ} \mathrm{C}$ for $30 \mathrm{~min}$, the reaction was stopped by adding $50 \mu \mathrm{l}$ $\mathrm{Na}_{2} \mathrm{CO}_{3}(0.5 \mathrm{M})$, and the released $p$-nitrophenol was measured spectrophotometrically at $405 \mathrm{~nm}$ by using a BioTekELx808 microplate reader (Winooski, VT, USA). The reaction mixture without enzyme was used as a blank. One unit $(\mathrm{U})$ of enzyme activity was defined as the amount of enzyme releasing $1 \mu \mathrm{mol} / \mathrm{min}$ of $p$-nitrophenyl under the assay conditions.

The effect of $\mathrm{pH}$ on PoAbf62A activity was determined at different pHs ranging from 2.0 to 11 at $37^{\circ} \mathrm{C}$ for $30 \mathrm{~min}$ using $p \mathrm{NP} \alpha \operatorname{Araf}(10 \mathrm{mM})$ as substrate. The $\mathrm{pH}$ stability was investigated under standard assay conditions following incubation of purified PoAbf62A for $24 \mathrm{~h}$ at $4{ }^{\circ} \mathrm{C}$ in the buffers without substrate. The optimum temperature was determined by measuring enzymatic activity at the optimal $\mathrm{pH}$ at temperatures ranging from 20 to $80{ }^{\circ} \mathrm{C}$. Thermostability was assessed by incubating the enzyme at different temperatures $\left(30-45^{\circ} \mathrm{C}\right)$ for up to $100 \mathrm{~min}$. Residual activities were assayed using $p \mathrm{NP} \alpha \mathrm{Ara} f$ as the substrate at $37{ }^{\circ} \mathrm{C}$ for $30 \mathrm{~min}$. The initial activity was defined as $100 \%$.

\section{Substrate specificity}

Substrate specificity was determined using various $p \mathrm{NP}$-glycosides as substrates, and the reaction was carried out in $200 \mu \mathrm{l}$ of $20 \mathrm{mM}$ Na-acetate buffer ( $\mathrm{pH} 4.5$ ) containing $10 \mathrm{mM}$ substrates and $2 \mu \mathrm{g}$ recombinant PoAbf62A. Hydrolytic activity towards polysaccharides was determined at $37{ }^{\circ} \mathrm{C}$ in Na-acetate buffer, $\mathrm{pH} 4.5$, with $0.5 \%$ (wt $/ \mathrm{vol})$ polysaccharides as substrates and $5 \mu \mathrm{g}$ PoAbf62A. After incubation for the appropriate reaction time, liberated reducing sugars were measured by the method of Somogyi (Somogyi 1952). To determine hydrolyzates of different arabinose-containing polysaccharides by HPAEC, the reaction mixture containing $50 \mu \mathrm{l}$ of a $4 \mathrm{mg} / \mathrm{ml}$ substrate solution, $140 \mu \mathrm{l}$ of $20 \mathrm{mM}$ Na-acetate buffer ( $\mathrm{pH} 4.5$ ), and $10 \mu \mathrm{l}$ of PoAbf62A $(100 \mu \mathrm{g} / \mathrm{ml})$ was performed for $12 \mathrm{~h}$ at $37{ }^{\circ} \mathrm{C}$, and the enzymatic productions were analyzed by using high-performance anion-exchange chromatography (HPAEC).

\section{Mode of action}

The mode of action of recombinant PoAbf62A was examined using sugar beet arabinan as substrate and analyzed by methylation and ${ }^{13} \mathrm{C}$ NMR. Sugar beet arabinan (20 mg) was treated with PoAbf62A for $12 \mathrm{~h}$ in conditions described above. The vacuum-dried sugar beet arabinan and enzymatic product $(10 \mathrm{mg})$ were methylated twice based on the method of Hakomori (Hakomori 1964). Methylated samples were hydrolyzed and reduced with sodium borohydride and acetylated. The partially methylated alditol acetates were analyzed by gas chromatography-mass spectrometry (GC-MS). In order to further assess the mode of action of PoAbf62A, the original sample and enzymatic product $(10 \mathrm{mg})$ were exchanged in $\mathrm{D}_{2} \mathrm{O}$ and analyzed by using ${ }^{13} \mathrm{C}$ NMR spectra on a Bruker Avance $600 \mathrm{MHz}$ spectrometer.

\section{HPAEC analysis}

Products were analyzed by HPAEC using a Dionex ICS 5000 system (Dionex Corp, Sunnyvale, CA, USA) with a CarboPac PA-200 analytical column $(3 \times 250 \mathrm{~mm}, \mathrm{P} /$ N062896) and pulsed amperometric detection. Samples were eluted at a flow rate of $0.5 \mathrm{ml} / \mathrm{min}$ with $0.05 \mathrm{M}$ 
$\mathrm{NaOH}$ for 10 min followed by a linear gradient from 0 to $0.1 \mathrm{M} \mathrm{Na}$-acetate in $0.05 \mathrm{M} \mathrm{NaOH}$ for $20 \mathrm{~min}$ and finally were eluted by $0.2 \mathrm{M} \mathrm{NaOH}$ and $0.5 \mathrm{M} \mathrm{Na}$-acetate for $10 \mathrm{~min}$.

\section{Methylation analysis}

Methylated samples of sugar beet arabinan and its products were analyzed by GC-MS with a Technologies 7890B GC and 5977B MSD equipped with a DB-1 capillary column $(0.25 \mathrm{~mm} \times 30 \mathrm{~m})$. The conditions of the GC column were as follows: initial temperature $120{ }^{\circ} \mathrm{C}$ for $1 \mathrm{~min}$, then $3{ }^{\circ} \mathrm{C} / \mathrm{min}$ to $210{ }^{\circ} \mathrm{C}$ for $2 \mathrm{~min}$, and then $10^{\circ} \mathrm{C} / \mathrm{min}$ to $260{ }^{\circ} \mathrm{C}$ for $4 \mathrm{~min}$; the injection temperature is $250{ }^{\circ} \mathrm{C}$. Nitrogen was used as a carrier gas and maintained at $1.2 \mathrm{ml} / \mathrm{min}$. The percentage of the methylated sugars was calculated as ratios of the peak areas.

\section{${ }^{13} \mathrm{C}$ NMR spectra}

${ }^{13} \mathrm{C}$ NMR spectra were obtained using a Bruker Avance $600 \mathrm{MHz}$ spectrometer (Bruker Inc., Rheinstetten, Germany) operating at $150 \mathrm{MHz}$ for carbon. Samples (10 mg) were dissolved in $\mathrm{D}_{2} \mathrm{O}(99.8 \%, 0.5 \mathrm{ml})$. Chemical shifts were given in ppm with acetone as the internal chemical shift reference.

\section{Nucleotide sequences}

The sequences for the 18S rDNA and PoAbf62A gene from P. oxalicum sp. 68 were submitted to GenBank with accession numbers GU078431 and MG874674, respectively.

\section{Results}

\section{Cloning, expression and purification of recombinant PoAbf62A}

The protein sequence of PoAbf62A from $P$. oxalicum (gene bank accession code EPS32936.1) predicted by Liu et al. (2013), contains 331 amino acids residues with a theoretical molecules mass of $35.9 \mathrm{kDa}$. Residues 35-329 have sequence similarity to GH family 62 (CAZy database: http://www.cazy.org/CAZY), and the sequences from 1 to 30 is predicted to be a signal sequence. Based on the nucleic acid sequence, the gene for removing the $\mathrm{N}$ terminal signal peptide was cloned into pET-32a $(+)$ (NdeI/EcoRI). The E. coli BL 21 (DE3) was chosen as host cell to produce recombinant PoAbf62A. Unfortunately, PoAbf62A formed inclusion bodies in solution because of its $N$-glycosylation sites. The recombinant plasmid (poabf62a-pPIC9K) was therefore transformed into GS115 cells by electroporation, and recombinant PoAbf62A was expressed under control of the alcohol oxidase promotor in GS115 and purified from the culture medium by ammonium sulfate precipitation and column chromatography. Using $p \mathrm{NP} \alpha \mathrm{Araf}$ as substrate, enzyme activity was determined by measuring the increase in reaction mixture at $405 \mathrm{~nm}$, and the results of purification are summarized in Table 1. Purified PoAbf62A was obtained with efficiently as detected by SDS-PAGE and use of artificial substrates.

\section{Biochemical characterization of recombinant PoAbf62A}

The hydrolytic activity of recombinant PoAbf62A was examined over a wide $\mathrm{pH}$ range from 2.0 to 11.0 using $p \mathrm{NP} \alpha$ Ara $f$ as substrate. At $\mathrm{pH} 4.5$, PoAbf62A exhibited its highest activity (Fig. 1a), as well as being quiet stable (Fig. 1b). At this $\mathrm{pH}$, the highest activity was observed at $35^{\circ} \mathrm{C}$ (Fig. 1c), whereas proteins stability was reduced at higher temperatures (Fig. 1d). Therefore, for efficiency in biotransformation and structural analysis, we chose the reaction conditions as $\mathrm{pH} 4.5$ and temperature of $35^{\circ} \mathrm{C}$.

\section{Substrate specificity of recombinant PoAbf62A on $p$ NP glycosides and carbohydrates}

Activity of PoAbf62A was tested by using different arylglycosides. Seven $p$ NP glycosides were chosen as substrates to analyze the activity of PoAbf62A. PoAbf62A exhibited high activity towards $p \mathrm{NP} \alpha \mathrm{Araf}$, whereas the enzyme showed little activity with $p N P \beta G l c$ and $p$ NP $\beta$ Xyl. PoAbf62A failed to hydrolyze $p$ NP $\alpha$ Ara $p$, $p \mathrm{NP} \alpha \mathrm{Gal}, \quad p \mathrm{NP} \beta \mathrm{Gal}$ and $p \mathrm{NP} \alpha \mathrm{Glc}$. Therefore, these results demonstrate that PoAbf62A from GH62 family is an $\alpha$-L-arabinofuranosidase. Using $p N P \alpha A r a f$ as a substrate, the kinetic parameters of purified recombinant PoAbf62A were determined. The $K_{\mathrm{m}}, V_{\max }$, and $k_{\text {cat }}$ values were $1.36 \pm 0.12 \mathrm{mM}, 0.49 \pm 0.01 \mu \mathrm{mol} / \mathrm{min} / \mathrm{mg}$, and $0.27 \pm 0.01 \mathrm{~s}^{-1}$, respectively. The catalytic efficiency $k_{\text {cat }} / K_{\mathrm{m}}$ was determined to be $0.20 \pm 0.02 \mathrm{mM}^{-1} \mathrm{~s}^{-1}$.

Furthermore, the specificity of PoAbf62A was assayed using different carbohydrates primarily those containing

Table 1 Summary of purification of recombinant PoAbf62A

\begin{tabular}{lccllll}
\hline Purification step & Volume $(\mathbf{m l})$ & Total protein $(\mathbf{m g})^{\mathbf{a}}$ & Activity $(\mathbf{U})^{\mathbf{b}}$ & Specific activity $(\mathbf{U} / \mathbf{m g})$ & Purification (fold) & Yield (\%) \\
\hline$\left(\mathrm{NH}_{4}\right)_{2} \mathrm{SO}_{4}$ & 20 & 16.7 & 3.8 & 0.56 & 1.0 & 100 \\
Phenyl Sepharose 6 Fast Flow (high & 2 & 2.2 & 1.75 & 0.80 & 1.43
\end{tabular}

sub)

a Protein was quantified according to the Bradford method using bovine serum albumin (BSA) as standard

b The activity was reported as activity on $p$ NPaAraf 

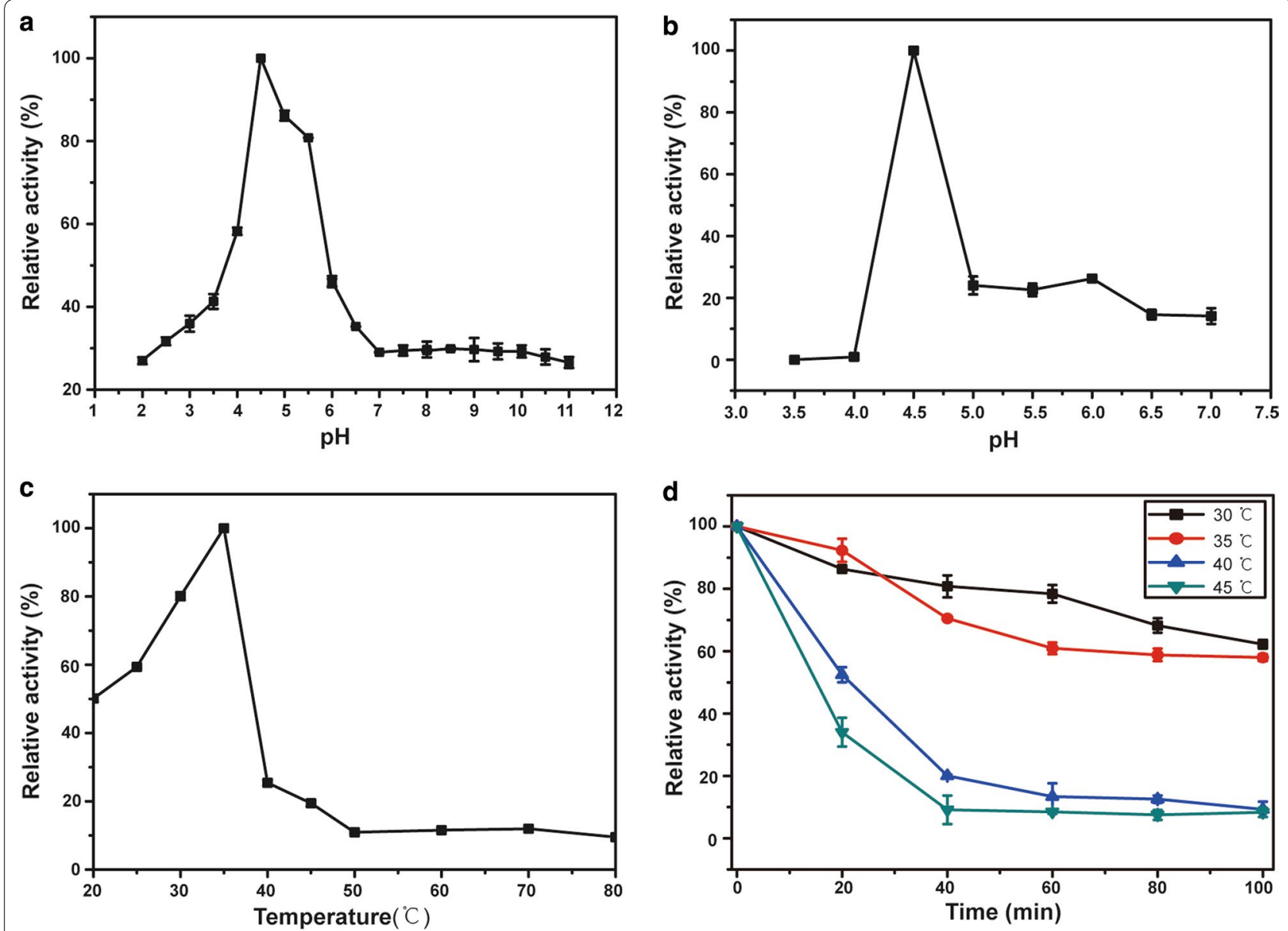

Fig. 1 pH and temperature profiles of the purified recombinant enzymes (PoAbf62A) The effect of pH on activity (a) and stability (b) of PoAbf62A used $p N P a A r a f$ as substrate; and the effect of temperature on activity (c) and stability (d) of PoAbf62A also used pNPaAraf as substrate. The activity of the enzyme before incubation was defined as 100\%. Results are presented as means \pm standard deviations $(n=3)$

arabinose, and detected by the method of Somogyi. The results showed that PoAbf62A could act on arabinosecontaining substrates such as sugar beet arabinan and wheat arabinoxylan which the release content of arabinose were 101 and $13 \mu \mathrm{g} / \mathrm{ml}$ after $12 \mathrm{~h}$ incubation with recombinant enzyme, whereas there was almost no impact on linear-1,5- $\alpha$-L-arabinan, arabinogalactan or xylan. The difference with sugar beet arabinan and linear$1,5-\alpha-\mathrm{L}$-arabinan is that the former contains branched $\alpha-1,2 / \alpha-1,3$-linked Araf, and we assumed that PoAbf62A specifically degrade $\alpha$-L-arabinofuranosyl side chains in sugar beet arabinan. In addition, PoAbf62A was effective on wheat arabinoxylan but no on xylan, meaning that PoAbf62A acts on $\alpha$-L-arabinofuranosyl of arabinoxylan, and not degrade the xylose backbone. Therefore, we assumed that PoAbf62A specifically acts on $\alpha-\mathrm{L}-$ arabinofuranosyl side chain.
HPAEC analysis of the degradation products by PoAbf62A

So far, our experiments indicated that PoAbf62A is active to artificial substrates and carbohydrates containing $\alpha$ - $\mathrm{L}$-arabinofuranosyl chain. To verify this hypothesis and analyze the products of PoAbf62A from different carbohydrates, we monitored the release of monosaccharide or oligosaccharide using HPAEC. As showed in Fig. 2, PoAbf62A could degrade sugar beet arabinan containing L-arabinofuranosyl side chains and released arabinose (Fig. 2a), whereas it did not work on linear1,5- $\alpha$-L-arabinan (Fig. 2b). Therefore, we concluded that PoAbf62A is an exo- $\alpha-\mathrm{L}$-arabinofuranosidase base on the degradetion of sugar beet arabinan that produces only monosaccharide. PoAbf62A also showed activity towards arabinoxylan $\alpha$-L-arabinofuranose (Fig. $2 \mathrm{~d}$ ), whereas it did not have the function on $\beta-1,4$ xylanase (Fig. 2c), because the production of arabinoxylan only had arabinose, and no xylose. Overall, PoAbf62A is an exo- $\alpha-\mathrm{L}-$ arabinofuranosidase that degrades branched arabinan 


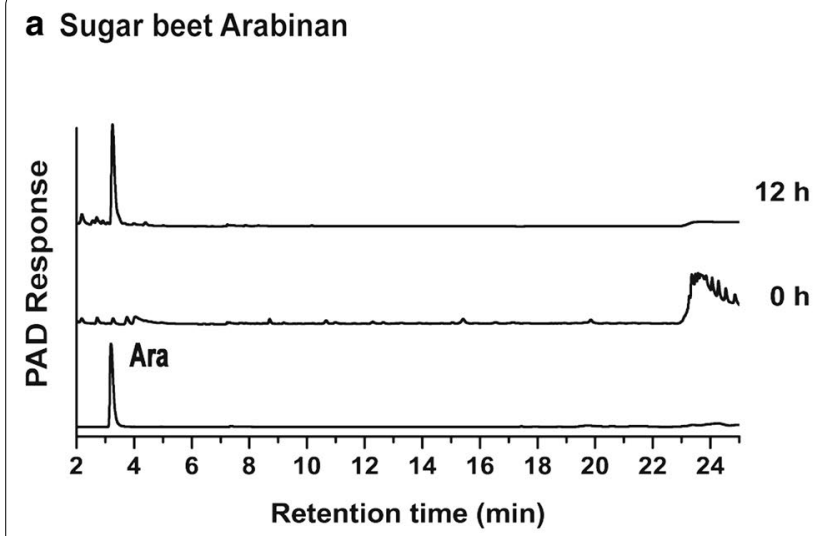

C Xylooligosaccharide

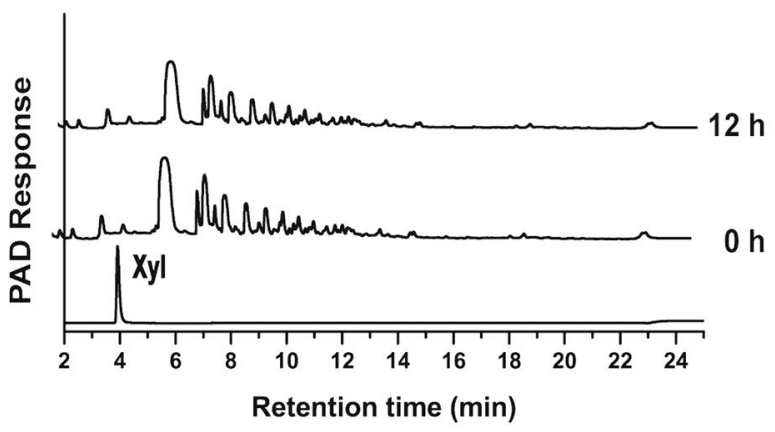

b Linear-1,5- $\alpha$-L-arabinan

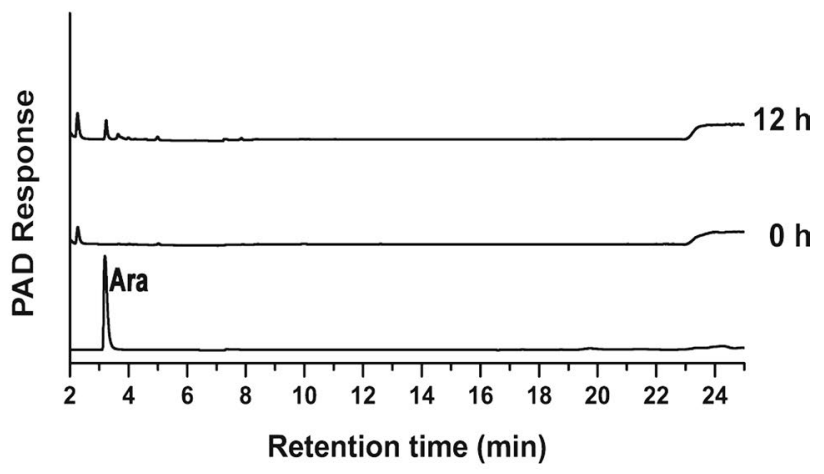

d Wheat arabinoxylan

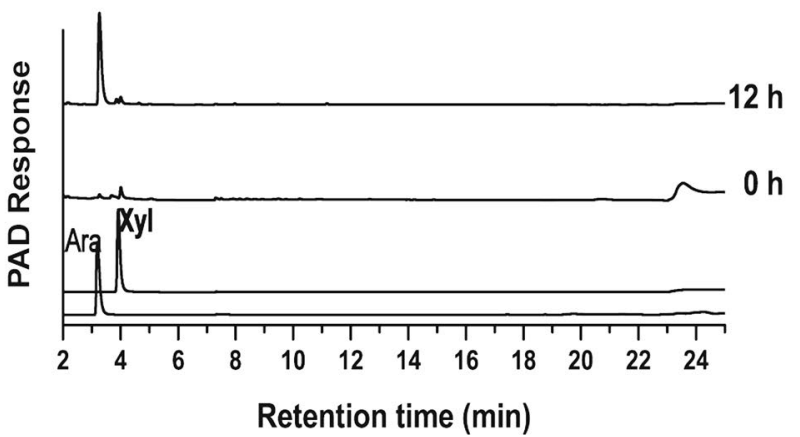

Fig. 2 HPAEC-PAD-200 analysis of degradation products of different polysaccharides by PoAbf62A. The reactions were incubated with recombinant enzyme at $37^{\circ} \mathrm{C}$ for $12 \mathrm{~h}$, and the experimental conditions are described in the text. The bottom of each graph was the standard sample of arabinose or xylose, and oligosaccharides of the reaction were detected by HPAEC at 0 and $12 \mathrm{~h}$, respectively

from sugar beets to produce arabinose; Moreover, it could also work on L-arabinofuranosyl side chains of arabinoxylan whose arabinoses are linked to C-2 or C-3 of arabinoxylan.

\section{Mode of action of PoAbf62A}

The side chains of sugar beet arabinan are decorated with single $\alpha-1,2$ or $\alpha-1,3-\mathrm{L}$-arabinofuranose, and some backbone residues are doubly substituted with both $\alpha-1,2$ and $\alpha-1,3$ side chains. To further explore the nature of glycosidic bond which PoAbf62A acts, we subjected sugar beet arabinan to methylation and ${ }^{13} \mathrm{C}$ NMR spectroscopy analysis post incubation with PoAbf62A. Methylation analysis indicated that the Ara residues mainly existed as terminal, 1,5-, 1,3,5-, 1,2,5- and 1,2,3,5-linked units, in addition containing a small amount of terminal, 1,6-linked Gal residues (Table 2). Among these residues, 1,5-linked Araf was approximately 39.7\%, with $28.5 \%$ being found at terminal positions. $24.0 \%$ of Araf residues were branched at the $\mathrm{O}-3$ position of backbone, and a few of the Ara $f$ residues were found as 1,2,5- and $1,2,3,5$,-linked units. It is very suitable as a substrate for the study of ABF. As shown in Table 2, the methylated products obviously changed following exposure to PoAbf62A. Five primary methylated products of Araf were detected, including 1,5- (58.9\%), 1,3,5- (1.3\%), 1,2,5(0.8\%) 1,2,3,5- (3.4\%) and terminal (7.8\%) linked Araf residues. It could be clearly seen that the content of terminal and 1,3,5-linked Araf decreased, while the content of 1,5-linked Ara $f$ increase, with the level of reduction being almost the same as the increase. The content of 1,2,5- and 1,2,3,5-linked Araf was not significantly modified. Taken together, this suggests that PoAbf62A can specifically hydrolyze $\alpha$-1,3-linked Araf chains present in sugar beet arabinan.

Furthermore, the structure of sugar beet arabinan and its enzymatic product were analysed by ${ }^{13} \mathrm{C} \mathrm{NMR}$, as exemplified in Fig. 3. The chemical shifts of major resonances were assigned based on literature values; the anomeric carbon signals of 1,3,5-linked Araf were identified at $106.97 \mathrm{ppm}$, and the C-3, C-4 of 1,3,5-linked Araf were found to resonate at $83.76 \mathrm{ppm}$ and $81.13 \mathrm{ppm}$, respectively (Cipriani et al. 2004). The variation in their ratio after hydrolysis indicated that the 1,3,5-linked Araf was 
Table 2 GC-MS analysis of the methylated products of sugar beet arabinan and its enzymatic product

\begin{tabular}{lllll}
\hline Methylated sugars & Type of linkage & Molar ratio (before) & Molar ratio (after) & Mass fragments (m/z) \\
\hline 1,4,5-Tri-O-actyl-2,3-di-O-methyl-arabinitol & 1,5-Linked Araf & 39.7 & 58.9 & $87,101,117,129,189$ \\
1,3,4-Tri-O-actyl-2,5-di-O-methyl-arabinitol & 1,3,5-Linked Araf & 24.0 & 1.3 & $85,99,117,127,159,201,261$ \\
1,2,4-Tri-O-actyl-3,5-di-O-methyl-arabinitol & 1,2,5-Linked Araf & 1.0 & 0.8 & $87,101,117,129,189,203$ \\
1,2,3,4,5-Penta-O-actyl-arabinitol & 1,2,3,5-Linked Araf & 4.2 & 3.4 & $85,115,127,145,187,233,289$ \\
1,4-Di-O-acetyl-2,3,5-tri-O-methyl-arabinitol & Terminal Araf & 28.5 & 7.8 & $87,101,117,129,161$ \\
$\begin{array}{l}\text { 1,5-Di-O-acetyl-1-deuterio-2,3,4,6-tetra-O-methyl- } \\
\text { D-galactitol }\end{array}$ & Terminal Galp & 1.3 & 1.2 & $71,87,101,117,129$ \\
$\begin{array}{l}\text { 1,5,6-Tri-O-acetyl-1-deuterio-2,3,4-tri-O-methyl- } \\
\text { D-galactitol }\end{array}$ & 1,6-Linked Galp & 1.5 & 1.0 & $87,101,117,129,143,161,189,233$ \\
\hline
\end{tabular}

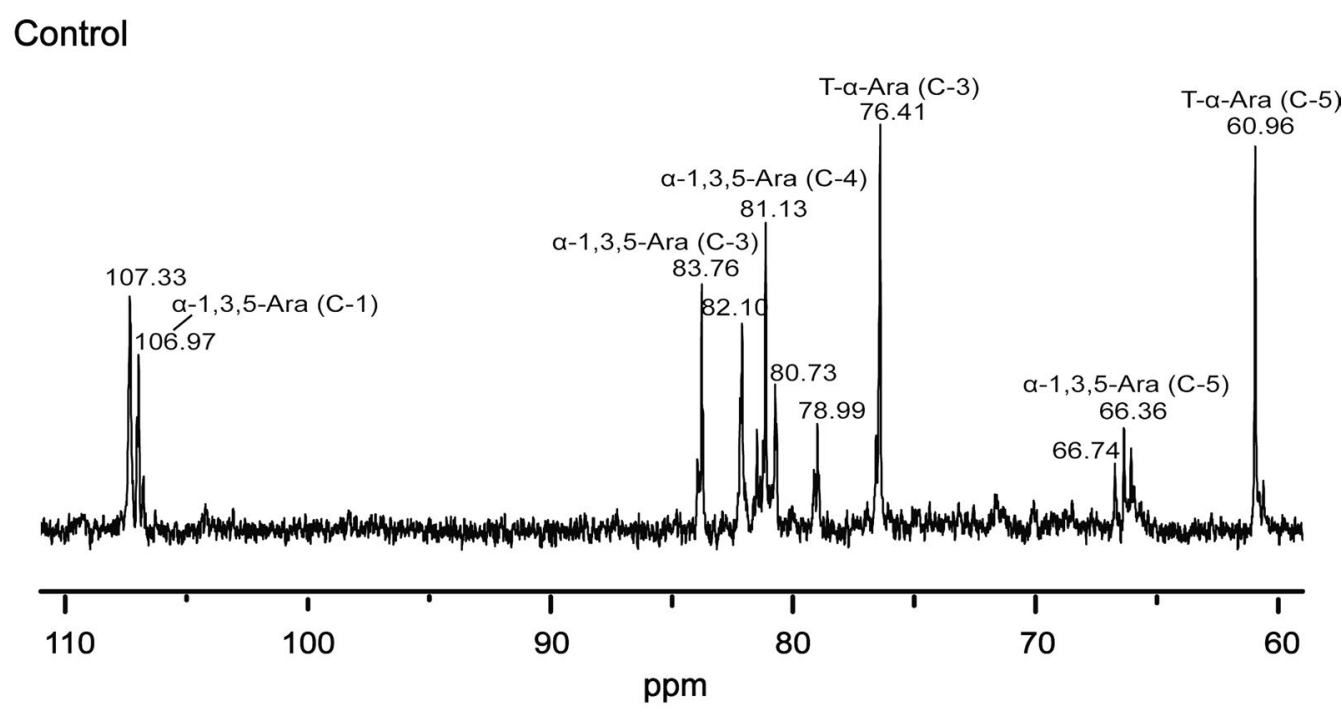

\section{PoAbf62A}

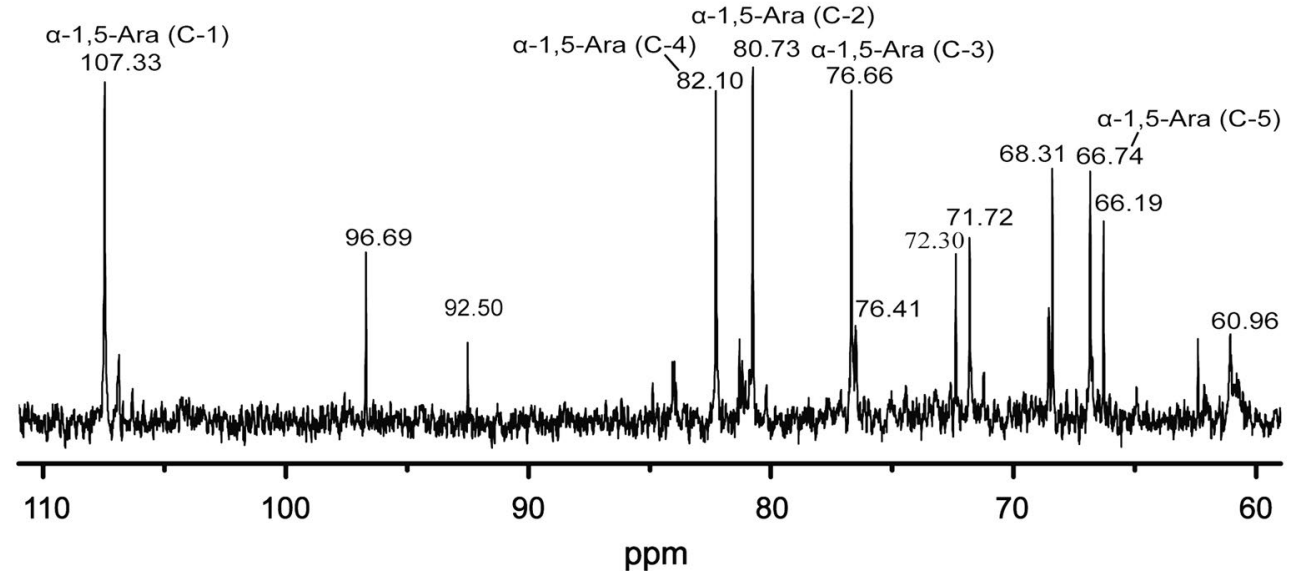

Fig. $3{ }^{13} \mathrm{C}$ NMR spectra of the production of sugar beet arabinan and sugar beet arabinan treated with the recombinant PoAbf62A. PoAbf62A $(50 \mathrm{mU})$ was incubated with $2 \mathrm{ml}$ of $0.5 \%$ sugar beet arabinan in $20 \mathrm{mM} \mathrm{Na}$-acetate buffer $(\mathrm{pH} 4.5)$ at $37^{\circ} \mathrm{C}$ for $12 \mathrm{~h}$, and the production was lyophilized and dissolved in $1 \mathrm{ml}$ of $\mathrm{D}_{2} \mathrm{O}$ for analysis 
cleaved and almost disappeared from there major polymers. Meanwhile, the carbon signal of terminal Araf was also significantly reduced. In contrast, the carbon signals of 1,5-linked Araf were increased as expected. Overall, these results support our proposal that PoAbf62A is an $\alpha-1,3$-arabinofuranosidase can specifically hydrolyze $\alpha-1,3$ linked Araf residues in sugar beet arabinan.

\section{Discussion}

Our present study reports on the ability of the recombinant enzyme PoAbf62A of the GH62 family from P. oxalicum sp. 68 to hydrolyze arabinose-containing polysaccharides. PoAbf62A exhibits a high specifity for $\alpha-1,3-\mathrm{L}$-arabinan and a much lower activity towards linear-1,5- $\alpha-\mathrm{L}$-arabinan. And the optimal reaction conditions of recombinant PoAbf62A was $\mathrm{pH} 4.5$ and temperature of $35{ }^{\circ} \mathrm{C}$, which showed better adaptability in an acid environment, likely because fungi prefer to grow under mildly acid conditions. This actually makes recombinant PoAbf62A more valuable in application with fruit juices and drinks that clarify under acid condition.

The GH62 family has been characterized with 22 enzymes of the 241 sequences annotated in the CAZy database (Wilkens et al. 2017), GH62 is the one that only contains ABFs in glycoside hydrolase families. The first reported ABFs of GH62 was TrAbf62A which was identified from Trichoderma reesei (Poutanen 1988). Like all characterized ABFs from the GH62 family, PoAbf62A can degrade arabinoxylan, and notably, PoAbf62A is also active on L-arabinan, there are only 11 ABFs in the GH62 family that exhibit activity on both arabinoxylan and L-arabinan (Siguier et al. 2014; McCleary et al. 2015), and others were also active on xylan or debranched arabinan. There are only two enzymes more active on sugar beet arabinan than on arabinoxylan (Couturier et al. 2011), and PoAbf62 was more active on sugar beet arabinan in this paper.

The GH62 family is suggested to be involved in plant cell wall penetration (Lanver et al. 2014) and synergistic effects from combination of various hydrolyzing enzymes against hemicelluloses show potential for their use in industrial biotechnology. The wealth of knowledge about the ABFs of the GH62 family helps to develop possible multiple applications. PoAbf62A showed more than $50 \%$ identity coverage with five $\alpha$-L-arabinofuranosidases from Penicillium which has been characterized as members of GH62 family. Research has shown that some GH62 ABFs hydrolyze both $\alpha-1,2$ and/or $\alpha-1,3$ linked Araf residues in arabinoxylan. ${ }^{1} \mathrm{H}$ NMR and hydrolytic fingerprinting revealed that $\alpha$ - $\mathrm{L}$-arabinofuranosidases from Penicillium chrysogenum (Sakamoto et al. 2011) and Penicillium funiculosum (De La Mare et al. 2013) cleave $\alpha-1,2-$ and $\alpha-1,3$-bonds that specifically link arabinofuranosyl moieties to single-substituted D-xylosyl residues in arabinoxylan. Meanwhile the enzymes from Podospora anserina, Ustilago maydis and Scytalidium thermophilum are able to efficiently remove the $\alpha-1,2$ and $\alpha-1,3-\mathrm{L}$-arabinosyl substituents from arabinoxylan which is supported by the crystallographic data (Siguier et al. 2014; Kaur et al. 2015). ABFII (Aspergillus fumigatus) and CcAbf62A (Coprinopsis cinerea) are specific for $\alpha-1,2-$ and/or $\alpha-1,3$ - bonds of arabinoxylan and sugar beet arabinan, while it did not further explain the mode of action by NMR or crystal (Pérez and Eyzaguirre 2016; Hashimoto et al. 2011). Besides, some ABFs from GH43 family could also release Araf in 1,2- or 1,3-linked groups in doubly substituted Xylp (Cartmell et al. 2011; Pouvreau et al. 2011). While none of them yet characterized ABFs from GH62 family have been shown to specifically hydrolyze $\alpha$-L-1,3 Araf from sugar beet arabinan. In our present study, we characterized PoAbf62A from $P$. oxalicum sp. 68 which is an exclusive $\alpha-1,3$-arabinofuranosidase determined by results of HPAEC, Methylation and NMR analysis. Our discovery thus enhances the potential of these ABFs for biotechnological applications, as well as the structural analysis of polysaccharides.

In conclusion, we cloned and characterized $\alpha-1,3-$ arabinofuranosidase PoAbf62A from $P$. oxalicum sp. 68, which is the first GH62 family $\alpha-1,3$-arabinofuranosidase reported from this strain. PoAbf62A can effectively hydrolyze $\alpha-1,3$-arabinofuranose groups in sugar beet arabinan and arabinoxylan, releasing arabinofuranose. The enzyme is an $\alpha-1,3$-arabinofuranosidase with specificity towards arabinose-containing polysaccharides. The discovery and characterization of the $\alpha-1,3-$ arabinofuranosidase PoAbf62A will provide a new tool as a biocatalysts to degrade and analyze the structure of plant cell walls. PoAbf62A will also be helpful for the application of P. oxalicum sp. 68 in the saccharification of lignocellulosic material.

\section{Abbreviations}

IPTG: isopropy- $\beta$-D-thiogalactoside; $D_{2} \mathrm{O}$ : heavy water; BGMY: buffered glycerol-complex medium; MD: minimal dextrose medium; G418: geneticin; LB: Luria-Bertani; OD: optical density.

\section{Authors' contributions}

ZYF and HYB conceived and designed the research. HYB, YXC, ZH, LJQ, LF, $C Y Y$ and WWY performed the experiments, HYB, YXC and $Z \mathrm{H}$ analyzed the data. ZYF and HYB wrote and edited the manuscript. All authors reviewed the manuscript. All authors read and approved the final manuscript.

\section{Acknowledgements \\ We are grateful to Prof. KH Mayo for critical reading and editing of our manuscript.}

\section{Competing interests}

The authors declare that they have no competing interests. 


\section{Availability of data and materials}

All data are shown in figures and tables within this article. Any material used in this study is available for research purposes upon request.

\section{Consent for publication}

Not applicable.

\section{Ethics approval and consent to participate}

This article does not contain any studies with human participants or animals performed by any of the authors.

\section{Funding}

This work was supported by the National Natural Science Foundation of China (No: 31470798 and 31770852), the Fundamental Research Funds for the Central Universities and Technology and Development Program of Jilin Province (Grant Numbers 20160520048JH).

\section{Publisher's Note}

Springer Nature remains neutral with regard to jurisdictional claims in published maps and institutional affiliations.

Received: 20 March 2018 Accepted: 24 March 2018

Published online: 02 April 2018

\section{References}

Altamimi MA, Palframan RJ, Cooper JM, Gibson GR, Rastall RA (2006) In vitro fermentation of sugar beet arabinan and arabino-oligosaccharides by the human gut microflora. J Appl Microbiol 100:407-414. https://doi. org/10.1111/j.1365-2672.2005.02780.x

Amore A, Amoresano A, Birolo L, Henrissat B, Leo G, Palmese A, Faraco V (2012) A family GH51 a-L-arabinofuranosidase from Pleurotus ostreatus: identification, recombinant expression and characterization. Appl Microbiol Biotechnol 94:995-1006. https://doi.org/10.1007/s00253-011-3678-4

Bradford MM (1976) A rapid and sensitive method for the quantitation of microgram quantities of protein utilizing the principle of protein-dye binding. Anal Biochem 72:248-254. https://doi org/10.1016/0003-2697(76)90527-3

Caffall KH, Mohnen D (2009) The structure, function, and biosynthesis of plant cell wall pectic polysaccharides. Carbohydr Res 344:1879-1900. https:// doi.org/10.1016/j.carres.2009.05.021

Cartmell A, McKee LS, Peña MJ, Larsbrink J, Brumer H, Kaneko S, Ichinose H, Lewis RJ, Viksø-Nielsen A, Gilbert HJ, Marles-Wright J (2011) The structure and function of an arabinan-specific a-1,2-arabinofuranosidase identified from screening the activities of bacterial $\mathrm{GH} 43$ glycoside hydrolases. J Biol Chem 286:15483-15495. https://doi.org/10.1074/jbc.M110.215962

Cipriani TR, Mellinger CG, Gorin PAJ, lacomini M (2004) An arabinogalactan isolated from the medicinal plant Maytenus ilicifolia. J Nat Prod 67:703-706. https://doi.org/10.1021/np034043f

Couturier M, Haon M, Coutinho PM, Henrissat B, Lesage-Meessen L, Berrin JG (2011) Podospora anserina hemicellulases potentiate the Trichoderma reesei secretome for saccharification of lignocellulosic biomass. Appl Environ Microbiol 77:237-246. https://doi.org/10.1128/AEM.01761-10

De La Mare M, Guais O, Bonnin E, Weber J, Francois JM (2013) Molecular and biochemical characterization of three $\mathrm{GH} 62$ a-L-arabinofuranosidases from the soil deuteromycete Penicillium funiculosum. Enzyme Microb Technol 53:351-358. https://doi.org/10.1016/j.enzmictec.2013.07.008

Gao D, Uppugundla N, Chundawat SP, Yu X, Hermanson S, Gowda K, Brumm P, Mead D, Balan V, Dale BE (2011) Hemicellulases and auxiliary enzymes for improved conversion of lignocellulosic biomass to monosaccharides. Biotechnol Biofuels 4:5. https://doi.org/10.1186/1754-6834-4-5

Gruppen H, Kormelink FJ, Voragen AG (1993) Water-unextractable cell wall material from wheat flour. 3. A structural model for arabinoxylan. J Cereal Sci 18:111-128. https://doi.org/10.1006/jcrs.1993.1040

Hakomori S (1964) A rapid permethylation of glycolipid, and polysaccharide catalyzed by methylsulfinyl carbanion in dimethyl sulfoxide. J Biochem 55:205-208. https://doi.org/10.1093/oxfordjournals.jbchem.a127869
Hashimoto K, Yoshida M, Hasumi K (2011) Isolation and characterization of CcAbf62A, a GH62 alpha-L-arabinofuranosidase, from the Basidiomycete Coprinopsis cinerea. Biosci Biotechnol Biochem 75:342-345. https://doi. org/10.1271/bbb.100434

Himmel ME, Ding SY, Johnson DK, Adney WS, Nimlos MR, Brady JW, Foust TD (2007) Biomass recalcitrance: engineering plants and enzymes for biofuels production. Science 315:804-807. https://doi.org/10.1126/ science. 1137016

Kaur AP, Nocek BP, Xu X, Lowden MJ, Leyva JF, Stogios PJ, Cui H, Leo RD, Powlowski J, Tsang A, Savchenko A (2015) Functional and structural diversity in $\mathrm{GH} 62$ a-L-arabinofuranosidases from the thermophilic fungus Scytalidium thermophilum. Microb Biotechnol 8:419-433. https://doi. org/10.1111/1751-7915.12168

Kormelink FJM, Gruppen H, Voragen AGJ (1993) Mode of action of (1-4)-beta-D-arabinoxylan arabinofuranohydrolase (AXH) and a-Larabinofuranosidases on alkali-extractable wheat-flour arabinoxylan. Carbohydr Res 249:345-353. https://doi.org/10.1016/0008-6215(93)84099-R

Laemmli UK (1970) Cleavage of structural proteins during the assembly of the head of bacteriophage T4. Nature 227:680-685. https://doi. org/10.1038/227680a0

Lanver D, Berndt P, Tollot M, Naik V, Vranes M, Warmann T, Münch K, Rössel $\mathrm{N}$, Kahmann R (2014) Plant surface cues prime Ustilago maydis for biotrophic development. PLoS Pathog 10:e1004272. https://doi.org/10.1371/ journal.ppat.1004272

Li J, Liu G, Chen M, Li Z, Qin Y, Qu Y (2013) Cellodextrin transporters play important roles in cellulose induction in the cellulolytic fungus Penicilium oxalicum. Appl Microbiol Biotechnol 97:10479-10488. https://doi. org/10.1007/s00253-013-5301-3

Liu G, Zhang L, Wei X, Zou G, Qin Y, Ma L, Li J, Zheng H, Wang S, Wang C, Xun L, Zhao GP, Zhou Z, Qu Y (2013) Genomic and secretomic analyses reveal unique features of the lignocellulolytic enzyme system of Penicillium decumbens. PLoS ONE 8:e55185. https://doi.org/10.1371/journal. pone.0055185

Lombard V, Ramulu HG, Drula E, Coutinho PM, Henrissat B (2014) The carbohydrate-active enzymes database (CAZy) in 2013. Nucleic Acids Res 42:D490-D495. https://doi.org/10.1093/nar/gkt1178

Maehara T, Fujimoto Z, Ichinose H, Michikawa M, Harazono K, Kaneko S (2014) Crystal structure and characterization of the glycoside hydrolase family 62 a-L-arabinofuranosidase from Streptomyces coelicolor. J Biol Chem 289:7962-7972. https://doi.org/10.1074/jbc.M113.540542

Margolles-Clark E, Tenkanen M, Nakari-Setälä T, Penttilä M (1996) Cloning of genes encoding alpha-L-arabinofuranosidases and beta-xylosidase from Trichoderma reesei by expression in Saccharomyces cerevisiae. Appl Environ Microbiol 62:3840-3846

McCleary BV, McKie VA, Draga A, Rooney E, Mangan D, Larkin J (2015) Hydrolysis of wheat flour arabinoxylan, acid-debranched wheat flour arabinoxylan and arabino-xylo-oligosaccharides by $\beta$-xylanase, $\mathrm{a}-\mathrm{L}-$ arabinofuranosidase and $\beta$-xylosidase. Carbohydr Res 407:79-96. https:// doi.org/10.1016/j.carres.2015.01.017

Numan MT, Bhosle NB (2006) a-L-Arabinofuranosidases: the potential applications in biotechnology. J Ind Microbiol Biotechnol 33:247-260. https:// doi.org/10.1007/s10295-005-0072-1

Pérez R, Eyzaguirre J (2016) Aspergillus fumigatus produces two arabinofuranosidases from glycosyl hydrolase family 62: comparative properties of the recombinant enzymes. Appl Biochem Biotechnol 179:143-154. https://doi.org/10.1007/s12010-016-1984-0

Pitson SM, Voragen AGJ, Beldman G (1996) Stereochemical course of hydrolysis catalyzed by arabinofuranosyl hydrolases. FEBS Lett 398:7-11. https:// doi.org/10.1016/S0014-5793(96)01153-2

Ponder GR, Richards GN (1997) Arabinogalactan from Western larch, part III: alkaline degradation revisited, with novel conclusions on molecular structure. Carbohydr Polym 34:251-261. https://doi.org/10.1016/ S0144-8617(97)00099-4

Poutanen K (1988) An a-L-arabinofuranosidase of Trichoderma reesei. J Biotechnol 7:271-281. https://doi.org/10.1016/j.biotechadv.2017.06.005

Pouvreau L, Joosten R, Hinz SWA, Gruppen H, Schols HA (2011) Chrysosporium lucknowense $\mathrm{C} 1$ arabinofuranosidases are selective in releasing arabinose from either single or double substituted xylose residues in arabinoxylan. Enzym Microb Technol 48:397-403. https://doi.org/10.1016/j. enzmictec.2011.01.004 
Saha BC (2000) a-L-Arabinofuranosidases: biochemistry, molecular biology and application in biotechnology. Biotechnol Adv 18:403-423. https://doi. org/10.1016/S0734-9750(00)00044-6

Sakamoto T, Kawasaki H (2003) Purification and properties of two type$\mathrm{B}$ a-L-arabinofuranosidases produced by Penicillium chrysogenum. Biochim Biophys Acta Gen Subj 1621:204-210. https://doi.org/10.1016/ S0304-4165(03)00058-8

Sakamoto T, Ogura A, Inui M, Tokuda S, Hosokawa S, Ihara H, Kasai N (2011) Identification of a GH62 alpha-L-arabinofuranosidase specific for arabinoxylan produced by Penicillium chrysogenum. Appl Microbiol Biotechnol 90:137-146. https://doi.org/10.1007/s00253-010-2988-2

Scigelova M, Singh S, Crout DHG (1999) Glycosidases-a great synthetic tool. J Mol Catal B Enzym 6:483-494. https://doi.org/10.1016/ S1381-1177(99)00012-0

Seiboth B, Metz B (2011) Fungal arabinan and L-arabinose metabolism. Appl Microbiol Biotechnol 89:1665-1673. https://doi.org/10.1007/ s00253-010-3071-8

Seri K, Sanai K, Matsuo N, Kawakubo K, Xue C, Inoue S (1996) L-Arabinose selectively inhibits intestinal sucrase in an uncompetitive manner and suppresses glycemic response after sucrose ingestion in animals. Metabolism 45:1368-1374. https://doi.org/10.1016/S0026-0495(96)90117-1

Siguier B, Haon M, Nahoum V, Marcellin M, Burlet-Schiltz O, Coutinho PM, Henrissat B, Mourey L, O'Donohue MJ, Berrin JG, Tranier S, Dumon C (2014) First structural insights into a-L-arabinofuranosidases from the two GH62 glycoside hydrolase subfamilies. J Biol Chem 289:5261-5273. https://doi. org/10.1074/jbc.M113.528133

Somogyi M (1952) Notes on sugar determination. J Biol Chem 195:19-23
Song L, Siguier B, Dumon C, Bozonnet S, O'Donohue MJ (2012) Engineering better biomass-degrading ability into a GH11 xylanase using a directed evolution strategy. Biotechnol Biofuels 5:3. https://doi. org/10.1186/1754-6834-5-3

Spagna G, Andreani F, Salatelli E, Romagnoli D, Casarini D, Pifferi PG (1998) Immobilization of the glycosidases: alpha-L-arabinofuranosidases and beta-D-glucopyranosidase from Aspergillus niger on a chitosan derivative to increase the aroma of wine. Part II. Enzyme Microb Technol 23:413421. https://doi.org/10.1016/S0141-0229(97)00249-4

Van Laere KM, Beldman G, Voragen AG (1997) A new arabinofuranohydrolase from Bifidobacterium adolescentis able to remove arabinosyl residues from double-substituted xylose units in arabinoxylan. Appl Microbiol Biotechnol 47:231-235. https://doi.org/10.1007/s002530050918

Vandermarliere E, Bourgois TM, Winn MD, Van Campenhout S, Volckaert G, Delcour JA, Strelkov SV, Rabijns A, Courtin CM (2009) Structural analysis of a glycoside hydrolase family 43 arabinoxylan arabinofuranohydrolase in complex with xylotetraose reveals a different binding mechanism compared with other members of the same family. Biochem J 418:39-47. https://doi.org/10.1042/BJ20081256

Wang WJ, Maigisondi G, Stogios PJ, Kaur A, Xu XH, Cui H, Turunen O, Savchenko A, Mastera ER (2014) Elucidation of the molecular basis for arabinoxylan-debranching activity of a thermostable family GH62 a-Larabinofuranosidase from Streptomyces thermoviolaceus. Appl Environ Microb 80:5317-5329. https://doi.org/10.1128/AEM.00685-14

Wilkens C, Andersen S, Dumon C, Berrin JG, Svensson B (2017) GH62 arabinofuranosidases: structure, function and applications. Biotechnol Adv 35:792-804. https://doi.org/10.1016/j.biotechadv.2017.06.005

\section{Submit your manuscript to a SpringerOpen ${ }^{\odot}$ journal and benefit from:}

- Convenient online submission

- Rigorous peer review

- Open access: articles freely available online

- High visibility within the field

- Retaining the copyright to your article

Submit your next manuscript at $\boldsymbol{\nabla}$ springeropen.com 